\title{
COVID-19 in Children: Clinical Approach and Management- Correspondence
}

To the Editor: We were pleased to read the well-written article in this journal entitled COVID-19 in children: clinical approach and management by Sankar J et al. [1]. We would like to commend the authors for a timely and succinct article on approach to management of pediatric COVID-19, providing essential and practical guidance to clinicians, and would like to comment on the management of COVID-19 in light of recent evidence, available studies and guidelines.

We are in the midst of an unprecedented global COVID-19 pandemic and though illness in children is usually mild, a small fraction can develop severe disease. The therapeutic approach for managing critically ill patients with Acute respiratory distress syndrome (ARDS)/ respiratory failure centers around lung protection with low tidal volumes, high positive end-expiratory pressures and fluid restriction. Anticoagulation is required in presence of high D-dimers or evidence of thromboembolism.

A variety of therapeutic agents for COVD-19 are under evaluation, with approximately 78 vaccine and 600 therapy related trials taking place worldwide. A recent study showed the antiviral Remdesivir, a nucleoside-analog that acts by inhibiting viral replication, to be effective in the majority of treated critically ill adult patients [2]. A recent multicenter expert pediatric guidance panel has recommended Remdesivir as the preferred antiviral agent in children if available [3]. Another therapeutic alternative for management in children is Hydroxychloroquine (HCQS), which can be used if Remdesivir is not available [3]. HCQS has been shown to be effective against SARS CoV-2 in in-vitro studies, considered relatively safe in children, and is also suggested by authors in the management approach. Of note, HCQS in combination with Azithromycin has been associated with greater risk of cardiac adverse effects and QTc prolongation, therefore, this combination is not recommended in children [3]. Other antivirals like 5-Fluorouracil, Ribavirin and Favipiravir, in treating COVID-19 by inhibiting the coronavirus' RNA proteins from making genomic copies of the novel coronavirus, are being studied as well. Although, Lopinavir/ Ritonavir was found to be of no benefit in one study as rightly noted by the authors [4], results of the World Health Organization SOLIDARITY trial evaluating these are awaited [5], and the pediatric panel neither recommends for /against its use [3]. The combination of Lopinavir/Ritonavir and Ribavirin is not recommended, given concern for serious adverse effects [3]. Convalescent plasma from recovered patients has been shown to be beneficial in critically ill patients [6] and given the accompanying cytokine storm with COVID19, IL-6 (interleukin-6) inhibitors like Tocilizumab are being increasingly utilized [7]. Other drugs like Famotidine are under study and low-dose steroids have been used in adults but studies pertaining to pediatric use are lacking.

As noted by the authors, the vast majority of pediatric patients with COVID-19 need only supportive care. As more scientific information becomes rapidly available, the decision to use therapeutic agents for critically ill children should be based on individual risk-benefit assessment, guided by the available evidence.

\footnotetext{
Ashlesha Kaushik ${ }^{1}$, Sandeep Gupta ${ }^{2}$ and Mangla Sood ${ }^{3}$

${ }^{1}$ Chief, Pediatric Infectious Diseases and COVID-19 Incident Command, Unity Point Health at St. Luke's Regional Medical Center; Department of Pediatrics, University of Iowa, Carver College of Medicine, Iowa, IA, USA; ${ }^{2}$ Director, Pulmonary Rehabilitation, COVID-19 Critical Care Incident Command; Division of Pulmonary and Critical Care, Unity Point Health at St. Luke's Regional Medical Center, Iowa, IA, USA; ${ }^{3}$ Department of Pediatrics, Former State Child Health Lead, Indira Gandhi Medical College, Shimla, Himachal Pradesh, India. E-mail: ashleshakaushik@gmail.com
} 


\section{References}

1. Sankar J, Dhochak N, Kabra SK, Lodha R. COVID-19 in children: clinical approach and management. Indian J Pediatr. 2020;87:433-42.

2. Grein J, Ohmagari N, Shin D, et al. Compassionate use of remdesivir for patients with severe covid-19. N Engl J Med. 2020. https://oi.org/ 10.1056/NEJMoa2007016. [Epub ahead of print].

3. Chiotos K, Hayes M, Kimberlin DW, et al. Multicenter initial guidance on use of antivirals for children with COVID-19/SARS-CoV-2. J Pediatr Infect Dis Soc. 2020 Apr 22. pii: piaa045; https://doi.org/10. 1093/jpids/piaa045. [Epub ahead of print]

4. Cao B, Wang Y, Wen D, et al. A trial of lopinavir-ritonavir in adults hospitalized with severe COVID-19. N Engl J Med. 2020;382:178799. https://doi.org/10.1056/NEJMoa2001282.

5. Kupferschmidt K, Cohen J. Race to find COVID-19 treatments accelerates. Science. 2020;367:1412-3.

6. Chen L, Xiong J, Bao L, Shi Y. Convalescent plasma as a potential therapy for COVID-19. Lancet Infect Dis. 2020;20:398-400.

7. Xu X, Han M, Li T, et al. Effective treatment of severe COVID-19 patients with tocilizumab. Proc Natl Acad Sci U S A. 2020;117:10970-5.

\section{Authors' Reply}

To the Editor: We thank Kaushik et al. for interest in our article "COVID-19 in children: Clinical approach and management" [1]. The authors provided an excellent summary of status of specific therapies in COVID-19 in children. As the disease is evolving and new evidence about drugs is published, management protocols are likely to evolve. We hereby summarize a few updates over our previously published article.

Though described as mild illness in children earlier, recently, a subset of children is seen to develop severe disease with multi-organs involved termed as "Multisystem Inflammatory Syndrome in Children" (MIS-C). MIS-C has clinical similarities to Kawasaki disease and toxic shock syndrome. Centers for Disease Control and Prevention defined MIS-C as patients (aged $<21 \mathrm{y})$ with fever, laboratory evidence of inflammation, severe symptoms with multiorgan involvement, and confirmed SARS-CoV-2 infection, and no alternative diagnostic possibility [2]. Common features described include gastrointestinal symptoms (diarrhea, abdominal pain), shock, myocardial dysfunction, coronary artery abnormalities, acute kidney injury, respiratory distress, and neurocognitive symptoms. Timely diagnosis and management of MIS-C with first-line therapy being immune modulation with human immunoglobulins or steroids is important, while some clinicians also administered aspirin and anticoagulants [2].

As pointed out by Kaushik et al., despite in-vitro suppression of SARS-CoV-2 by various drugs, most clinical studies have not found significant benefit with therapy. As previously described, viral titers are highest in respiratory tract in initial phase of illness (flu like illness), antiviral therapies are likely to have maximum efficacy when started within first 1-2 d of illness [3]. Remdesivir has been shown to decrease duration of hospitalization in adults with COVID-19 [4]. Among the options available, Remdesivir could be the first choice drug for COVID-19 in children. But in regions where Remdesivir is not available, either Lopinavir/Ritonavir or Hydroxychloroquine (or Chloroquine) maybe used in children with severe disease (preferably as a part of clinical trial). Recent evidence points towards lack of efficiency and significant arrhythmogenic side-effects of Hydroxychloroquine and Chloroquine (especially in combination with macrolides) in adults; these drugs should be used with caution [5]. But certain high-risk groups (infants and children with co-morbidities) could benefit from early anti-viral therapies (preferably as a part of clinical trial; Remdesivir or Lopinavir/ Ritonavir or Hydroxychloroquine, in that order of preference) [3].

Understanding of the pathogenesis of respiratory failure is also evolving. Though virus-induced diffuse alveolar damage is the major pathology, a recent report demonstrated frequent endothelialitis and thrombosis in alveolar capillaries in COVID-19 autopsies in adults [6]. Silent hypoxia is being observed in a fraction of patients, probably due to vascular involvement, pointing to need for routine oxygen saturation $\left(\mathrm{SpO}_{2}\right)$ monitoring in patients who are apparently well [7]. Also, COVID-19 related acute respiratory distress syndrome (ARDS) has been classified as L-phenotype (low elastance, high compliance) and H-phenotype (high elastance, low compliance). L-phenotype usually has normal lung compliance, and can be managed with high flow, high $\mathrm{FiO}_{2}$ therapies (such as high flow nasal canula), non-invasive ventilation or mechanical ventilation (but lower positive end-expiratory pressure, PEEP). H-phenotypes should be managed with traditional ARDS protocols (high PEEP, low tidal volume) [8]. Prone positioning in non-intubated patients may be beneficial in a subset of patients, but potential harm in infants due to risk of sudden infant death should be kept in mind [9].

We had suggested exclusive breastfeeding for all neonates born to COVID-19 mothers [1]. Recent American Association of Pediatrics guidelines suggest that breastmilk should be preferred feed for the baby while decision to directly breastfeed or feeding with expressed breastmilk should be based on choice of mother and family members. Baby should preferably be nursed with a designated COVID-19 negative care-giver, whenever feasible, till mother is non-infective [10]. Regional guidelines regarding breastfeeding should be followed.

Nitin Dhochak and Rakesh Lodha

Department of Pediatrics, AIIMS, New Delhi, India. E-mail: rlodha1661@gmail.com 


\section{References}

1. Sankar J, Dhochak N, Kabra SK, Lodha R. COVID-19 in children: Clinical approach and management. Indian J Pediatr. 2020;87:433-42.

2. Experts Shed More Light on COVID-19-Related Inflammatory Syndrome in Children. Available at: https:/www.aappublications. org/news/2020/05/20/covid19inflammatorysyndrome 052020 . Accessed 22 May 2020.

3. Dhochak N, Singhal T, Kabra SK, Lodha R. Pathophysiology of COVID-19: Why children fare better than adults? Indian J Pediatr. 2020; https://doi.org/10.1007/s12098-020-03322-y.

4. Beigel JH, Tomashek KM, Dodd LE, et al. Remdesivir for the treatment of COVID-19 -Preliminary report. N Engl J Med. 2020; https:// doi.org/10.1056/NEJMoa2007764.

5. Mehra MR, Desai SS, Ruschitzka F, Patel AN. Hydroxychloroquine or chloroquine with or without a macrolide for treatment of COVID-19: A multinational registry analysis. Lancet. 2020; https://doi.org/10.1016/ S0140-6736(20)31180-6.

6. Ackermann M, Verleden SE, Kuehnel M, et al. Pulmonary vascular endothelialitis, thrombosis, and angiogenesis in COVID-19. N Engl J Med. 2020; https://doi.org/10.1056/NEJMoa2015432.
7. "Silent Hypoxia" may be Killing COVID-19 Patients. But There's Hope. Available at: https://www.livescience.com/silent-hypoxiakilling-covid-19-coronavirus-patients.html. Accessed 22 May 2020.

8. Marini JJ, Gattinoni L. Management of COVID-19 respiratory distress. JAMA. 2020; https://doi.org/10.1001/jama.2020.6825.

9. Elharrar X, Trigui Y, Dols A-M, et al. Use of prone positioning in non-intubated patients with COVID-19 and hypoxemic acute respiratory failure. JAMA. 2020; https://doi.org/10.1001/jama.2020. 8255.

10. Breastfeeding Guidance Post Hospital Discharge for Mothers or Infants with Suspected or confirmed SARS-Co V-2 Infection. Available at: https://services.aap.org/en/pages/2019-novelcoronavirus-covid-19-infections/breastfeeding-guidance-posthospital-discharge/. Accessed 22 May 2020.

Publisher's Note Springer Nature remains neutral with regard to jurisdictional claims in published maps and institutional affiliations. 